\title{
Snapshot of Phylogenetic Groups, Virulence, and Resistance Markers in Escherichia coli Uropathogenic Strains Isolated from Outpatients with Urinary Tract Infections in Bucharest, Romania
}

\author{
Violeta Corina Cristea, ${ }^{1,2}$ Irina Gheorghe, ${ }^{3,4}$ Ilda Czobor Barbu, ${ }^{3,4}$ \\ Laura Ioana Popa, ${ }^{3,4}$ Bogdan Ispas, ${ }^{3,4}$ Georgiana Alexandra Grigore, ${ }^{3,4}$ \\ Irina Bucatariu, ${ }^{3,4}$ Gabriela Loredana Popa, ${ }^{2}$ Maria-Cristina Angelescu, ${ }^{1}$ \\ Alexandra Velican, ${ }^{3,4}$ Luminita Marutescu, ${ }^{3,4}$ Marcela Popa, \\ Mariana Carmen Chifiriuc, ${ }^{3,4}$ and Ioan Mircea Popa $\mathbb{D}^{2,5}$ \\ ${ }^{1}$ Central Laboratory Synevo-Medicover, Bucharest, Romania \\ ${ }^{2}$ University of Medicine and Pharmacy Carol Davila, Bucharest, Romania \\ ${ }^{3}$ Department of Microbiology and Immunology, Faculty of Biology, University of Bucharest, Romania \\ ${ }^{4}$ Research Institute of the University of Bucharest (ICUB), Bucharest, Romania \\ ${ }^{5}$ National Medico-Military Institute for Research and Development Cantacuzino, Bucharest, Romania
}

Correspondence should be addressed to Ioan Mircea Popa; mircea.ioan.popa@gmail.com

Received 6 January 2019; Revised 1 April 2019; Accepted 30 April 2019; Published 20 May 2019

Guest Editor: Tomasz M. Karpiński

Copyright (C) 2019 Violeta Corina Cristea et al. This is an open access article distributed under the Creative Commons Attribution License, which permits unrestricted use, distribution, and reproduction in any medium, provided the original work is properly cited.

Background. Urinary tract infections (UTIs) caused by Uropathogenic Escherichia coli (UPEC) are among the most common infections worldwide, including Romania. To the best of our knowledge, this is the first study performed on a significant number of community-acquired (CA) UPEC strains isolated from Romanian outpatients, aiming to evaluate and establish potential correlations among the phylogenetic groups (PG), resistance profiles, and the virulence factors (VF) genes of the CA-UPEC isolates. Materials/Methods. The present study was performed on a total of 787 UPEC nonrepetitive isolates consecutively isolated during one month from outpatients with CA-UTIs, visiting one of the biggest laboratories in Bucharest, Romania, receiving patients from all over the country. The strains identification was performed by MALDI TOF and the susceptibility patterns were tested using Microscan according to CLSI guidelines. PCR assays were performed to detect the presence of different VFs (fimH gene encoding for type 1 fimbriae, $a f a B C$ for A fimbriae, $s f a D E$ for S fimbriae, KpsMTII for capsule, $h l y A$ for haemolysin A, $h l y D$ for haemolysin $\mathrm{D}$, and $c n f-1$ for tumor necrosis factor), the phylogenetic groups (PG) A, B1, B2, and D, and the extended spectrum beta-lactamases (ESBLs) genes. Results. The 787 CA-UPEC strains were isolated predominantly from female patients $(90.95 \%)$ of $>30$ years ( $74 \%)$. The resistance rates were $47.52 \%$ for ampicillin, $41.16 \%$ for tetracycline, $24.39 \%$ for cotrimoxazole, $19.18 \%$ for amoxicillin-clavulanic acid, $15.50 \%$ for cefazolin, $14.99 \%$ for ciprofloxacin, and $14.86 \%$ for levofloxacin; $35.19 \%$ of the investigated strains were MDR and 9.03\% ESBL producers (from which $42.25 \%$ were positive for blaCTX-M, 38.02\% for blaTEM, and 19.71\% for blaSHV). FimH was the most frequent virulence gene (93.90\%) followed by hlyD (44.34\%); afaBC (38.24\%); KpsMTII (32.65\%); sfaDE (23.88\%); hlyA (12.45\%); and $c n f-1$ (7.75\%). The distribution of the analyzed UPEC strains in phylogenetic groups was different for non-MDR and MDR strains. Overall, $35 \%$ of the strains belonged to the phylogenetic group B2 (harboring the yjaA gene); $27 \%$ to group B1 (confirmed by the presence of the TspE4C2 fragment); 16\% to group D; and 22\% to group A. The CA-UPEC strains included in PG $\mathrm{B} 1$ and PG B2 proved to be the most virulent ones, the number of strains carrying multiple VFs $(>3)$ being significantly larger as compared to strains belonging to PG A and PG D) $(p<0,0001)$. The presence of one or two ESBL genes was significantly associated ( $\mathrm{p}=0.0024$ ) with PGs A and D. Conclusions. Our findings showed that the community UPEC strains circulating in Bucharest, Romania, belong predominantly to group B2 and $>90 \%$ harbored the fim $H$ gene. High MDR resistance rates were observed, as well 
as extended VF profiles, highlighting the importance of this type of studies for improving the epidemiological surveillance and the therapeutic or prophylactic management of the respective infections, in the context of antibiotic resistance emergence.

\section{Background}

Urinary tract infection (UTI) caused by Uropathogenic Escherichia coli (UPEC) is the most common communityacquired (CA) and nosocomial infection $[1,2]$ and represents an important worldwide health problem, leading to considerable morbidity costs [3-5]. E. coli is the leading cause of UTIs, being responsible for $75-90 \%$ of UTIs in ambulatory patients [6]. These isolates encode different virulence factors (VFs), like toxins, capsules, invasins, and adhesins, which are contributing to the UPEC strains pathogenicity and consequently, to the severity of the produced UTI [7]. Moreover, the emergence of multidrug-resistant (MDR) UPEC strains is currently leading to major difficulties in treating the infected patients [8-10].

UPEC strains have been classified into several main phylogenetic groups (PG) (A, B1, B2, C, D, E, and F) and one Escherichia cryptic clade I, based on the combination of four genetic markers: $\operatorname{arpA}$, chuA, yjaA, and the DNA fragment TspE4C2 [11]. UPEC strains usually belong to group $\mathrm{B} 2$ and to a lesser extent, to group $\mathrm{D}$, whereas commensal strains belong to groups A and B1 [12]. Among B2 strains, E. coli sequence type 131 (ST131) is considered an important emerging pathogen, harboring numerous resistance and VF genes [13]. Strains belonging to this group are resistant to most $\beta$-lactam antibiotics, mediated by the production of extended spectrum $\beta$-lactamases (ESBLs). ESBLs are plasmid-encoded enzymes which confer resistance to penicillins, broad-spectrum cephalosporins, and monobactams, but not to cephamycins and carbapenems. Moreover it has been revealed that ESBL-producing isolates show coresistance to aminoglycosides, quinolones, tetracyclines, nitrofurantoin, and trimethoprim-sulfamethoxazole [14]. The MDR phenotype is due to the presence of large plasmids, which commonly carry resistance genes for $\beta$-lactams, quinolones, aminoglycosides, and cotrimoxazole. The most common $\beta$ lactamases in E. coli strains are TEM, SHV, and CTX-M types [14]. Most ST131 strains belong to the O25:H4 serotype, with the specific O25b type. However, ST131 strains with serotype O16:H5 have been recently identified, as well as some others that are nontypeable for $\mathrm{O}$ and $\mathrm{H}$ antigens [15]. UPEC clones ST69, ST95, and ST73 are also frequent causes of UTIs and bloodstream infections. A study performed in Brazil showed that UTIs in men were more frequently caused by PG B2 isolates, harboring an extended VFs genes profile [12]. A study performed in Iran on 232 UPEC strains revealed that the most frequent PG was D (both for hospital and CA infections (58\%), exhibiting the highest number of $\mathrm{VF}$ and resistance markers [16].

The aim of the study was to characterize the resistance and virulence profiles of recently isolated UPEC strains from outpatients visiting Synevo Central Laboratory, Medicover, in Bucharest, south Romania, and to establish potential correlations among PG, resistance, and VF genes profiles of the analyzed strains. To the best of our knowledge, this is the first study performed on a significant number of UPEC strains isolated from CA-UTIs in Romania.

\section{Results and Discussion}

The 787 UPEC strains were isolated predominantly from female patients $(90.95 \%)$ and the distribution on age groups was the following: $0-16$ years $(8.66 \%)$; $16-30$ years $(16.94 \%)$; $30-50$ years $(35.79 \%)$, and $50-90$ years $(38.29 \%)$.

2.1. Phylogenetic Group Distribution of UPEC Strains. The PG analysis of the UPEC strains $[17,18]$ showed that $35 \%$ of the strains belonged to $\mathrm{B} 2$ (harboring the $y j a A$ gene); $27 \%$ to $\mathrm{B} 1$ (confirmed by the presence of TspE4 C2 gene); 16\% to D and $22 \%$ to $\mathrm{A}$. The obtained results are very close to those reported from Nüesch-Inderbinen et al., in UPEC strains isolated from community-acquired UTI in Switzerland [19].

2.2. Antibiotic Resistance Profiles of UPEC Strains. In this study, the prevalence of antimicrobial resistance markers was relatively high for drugs commonly used as emergency therapy in the treatment of UTIs, such as ampicillin (47.52\%), tetracycline $(41.16 \%)$, trimethoprim-sulfamethoxazole (cotrimoxazole) (24.39\%), amoxicillin-clavulanic acid (19.18\%), cefazolin (15.50\%), and fluoroquinolones (14.99\% for ciprofloxacin and $14.86 \%$ for levofloxacin). Moreover, $35.19 \%$ of the UPEC strains were MDR, according to Magiorakos et al., 2012 [20] criteria. Lower resistance percentages were recorded for aztreonam, cefepime, ceftriaxone, piperacillintazobactam, and gentamicin (Table 1), in contrast with the study performed by Lavigne et al., in 2016, which reported higher resistance percentages for amoxicillin, amoxicillinclavulanic acid, nalidixic acid, ciprofloxacin, and nitrofurantoin and for cotrimoxazole in E. coli strains from CA-UTI.

Out of the total of the analyzed strains, 71 isolates (9.03\%) were resistant to third-generation cephalosporins, all of them being positive for the investigated ESBL genes, as follows: blaCTX-M (42.25\%) and blaTEM (38.02\%) and for blaSHV (19.71\%) (Table 2). Regarding the resistance profiles of the ESBL strains, they were highly resistant to ampicillin (100\%), aztreonam (98.59\%), cefepime (98.59\%), ceftriaxone $(91.59 \%)$, cefazolin $(87.32 \%)$, tetracycline $(73.23 \%)$, ciprofloxacin (71.83\%), and levofloxacin (70.42\%) (Table 2).

A study performed in Algeria, aiming to investigate antibiotic resistance and VF in 150 nonrepetitive CA-UPEC isolates has revealed a MDR rate of $46.7 \%$ [21]. The detected bla genes were blaTEM $(96.8 \%$ of amoxicillin-resistant isolates), blaCTX-M-15 (4\%), blaAmpC (4\%), blaSHV-2a, blaTEM-4, blaTEM-31, and blaTEM-35 (0.7\%) [21].

Our study has revealed that the MDR strains were classified in PG D (44.71\%), followed by PG A (40.58\%), PG B1 (32.71\%), and PG B2 (29.71\%), while the non-MDR strains were predominantly associated with PG B2, followed by B1, and to a lesser extent with the other two PGs (Figure 1). A 
TABLE 1: Antibiotic resistance phenotypes of E. coli strains isolated from CA-UTIs in Romania.

\begin{tabular}{|c|c|c|c|c|c|}
\hline Antibiotic & GR A & GR B1 & GR B2 & GR D & Number (percentage) \\
\hline AMP & $90(52.94 \%)$ & $98(45.16 \%)$ & $125(45.28 \%)$ & $66(53.65 \%)$ & $374(47.52 \%)$ \\
\hline TET & $75(44.11 \%)$ & $91(41.93 \%)$ & $98(35.50 \%)$ & $61(49.59 \%)$ & $324(41.16 \%)$ \\
\hline SXT & $43(25.29 \%)$ & $52(23.96 \%)$ & $60(21.73 \%)$ & $41(33.33 \%)$ & $192(24.39 \%)$ \\
\hline $\mathrm{AMC}$ & $38(22.35 \%)$ & $41(18.89 \%)$ & $55(19.92 \%)$ & $24(19.51 \%)$ & $151(19.18 \%)$ \\
\hline CFZ & $35(20.58 \%)$ & $29(13.36 \%)$ & $42(15.21 \%)$ & $17(13.82 \%)$ & $122(15.50 \%)$ \\
\hline CIP & $34(20 \%)$ & $38(17.51 \%)$ & $28(10.14 \%)$ & $18(14.63 \%)$ & $118(14.99 \%)$ \\
\hline LEV & $34(20 \%)$ & $38(17.51 \%)$ & $28(10.14 \%)$ & $18(14.63 \%)$ & $117(14.86 \%)$ \\
\hline ATM & $16(9.41 \%)$ & $21(9.67 \%)$ & $27(9.78 \%)$ & $11(8.94 \%)$ & $79(10.03 \%)$ \\
\hline FEP & $16(9.41 \%)$ & $21(9.67 \%)$ & $26(9.42 \%)$ & $10(8.13 \%)$ & $73(9.27 \%)$ \\
\hline $\mathrm{CRO}$ & $16(9.41 \%)$ & $21(9.67 \%)$ & $26(9.42 \%)$ & $10(8.13 \%)$ & $72(9.14 \%)$ \\
\hline $\mathrm{TZP}$ & $17(10 \%)$ & $11(5.06 \%)$ & $16(5.79 \%)$ & $3(2.43 \%)$ & $47(5.97 \%)$ \\
\hline $\mathrm{CN}$ & $12(7.05)$ & $6(2.76 \%)$ & $10(3.62 \%)$ & $7(5.69 \%)$ & $34(4.32 \%)$ \\
\hline NIT & $5(2.94 \%)$ & $8(3.68 \%)$ & $7(2.53 \%)$ & $4(3.25 \%)$ & $24(3.04 \%)$ \\
\hline IMP & 0 & 0 & 0 & $1(0.81 \%)$ & $1(0.12 \%)$ \\
\hline MEM & 0 & 0 & 0 & 0 & 0 \\
\hline ETP & 0 & 0 & 0 & 0 & 0 \\
\hline
\end{tabular}

AMP, ampicillin; TET, tetracycline; SXT, trimethoprim-sulfamethoxazole; AMC, amoxicillin-clavulanic acid; CFZ, cefazolin; CIP, ciprofloxacin; LEV, levofloxacin; ATM, aztreonam; FEP, cefepime; CRO, ceftriaxone; TZP, piperacillin-tazobactam; CN, gentamicin; NIT, nitrofurantoin; IMP, imipenem; MEM, meropenem; ETP, ertapenem.

TABLE 2: Antibiotic resistance phenotypes of ESBL strains.

\begin{tabular}{lc}
\hline Antibiotic & Number (percentage) \\
\hline AMP & $71(100 \%)$ \\
ATM & $70(98.59 \%)$ \\
FEP & $70(98.59 \%)$ \\
CRO & $65(91.59 \%)$ \\
CFZ & $62(87.32 \%)$ \\
TET & $52(73.23 \%)$ \\
CIP & $51(71.83 \%)$ \\
LEV & $50(70.42 \%)$ \\
SXT & $34(47.89 \%)$ \\
AMC & $32(45.07 \%)$ \\
TZP & $17(23.94 \%)$ \\
NIT & $6(8.45 \%)$ \\
\hline
\end{tabular}

different distribution was recorded for the ESBL-producing strains, which belonged to PG B1 (10.13\%); PG A (9.41\%); PG B2 (8.33\%), and PG D (8.13\%). However, the statistical analyses did not reveal any statistical significance of the correlation between the antibiotic resistance and E. coli phylogenetic groups.

In other studies, the CA-UPEC isolates belonged to phylogroups $\mathrm{B} 2+\mathrm{D}(50 \%), \mathrm{A}+\mathrm{B} 1(36 \%)$, and $\mathrm{F}+\mathrm{C}+\mathrm{Clade} \mathrm{I}$ (13\%). Most of D (72.2\%) and $38.6 \%$ of B2 isolates were MDR and harbored the most extended VFs profiles [21].

2.3. Virulence Profiles of UPEC Strains. Regarding the virulence markers detected in the analyzed UPEC strains, the fimH gene was the most encountered VF (93.90\%) followed by $h l y D$ (44.34\%); afaBC (38.24\%); KpsMTII (32.65\%); sfaDE (23.88\%); hlyA (12.45\%); and cnf-1 (7.75\%) (Table 3).

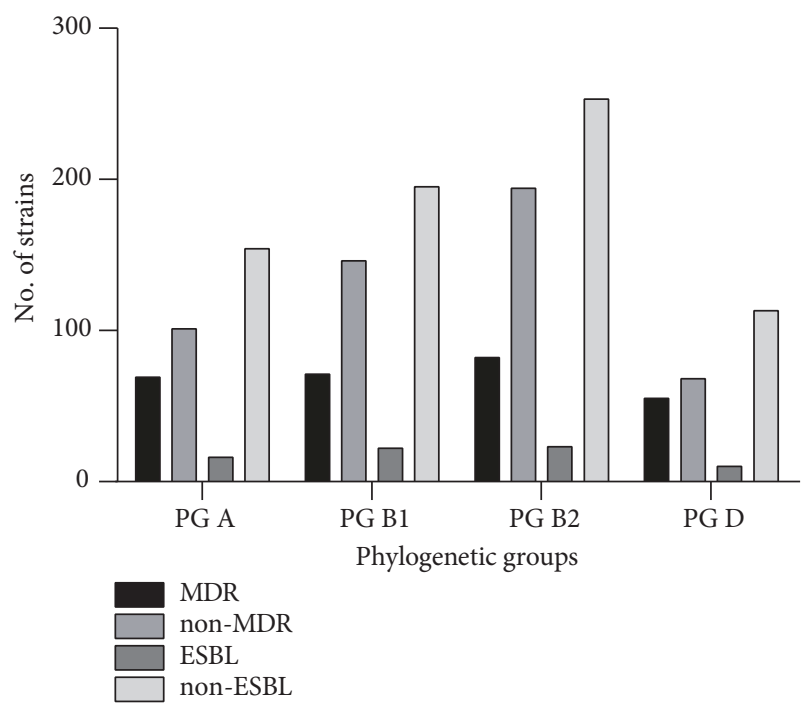

FIGURE 1: The distribution of ESBL and MDR isolates by phylogenetic groups.

Distribution of the analyzed strains harboring different VF in PGs revealed a relatively equal distribution among the PG for fimH, while others ( $h l y A, a f a B C, k p s M T I I, s f a D E$, and cnf-1) were significantly associated with certain PGs (Figure 2 and Table 3).

Regarding the correlation between the pathogenicity level (the number of VF genes) and the phylogenetic groups, the strains belonging to $\mathrm{PG} \mathrm{D}(\mathrm{n}=123$ strains) revealed the following VF genes profiles: $5 \mathrm{VFs}$ in $4.06 \%$ of the investigated strains; 4 VFs (fim H, hlyD, sfaDE cnf-1, and $h l y A$ ) in $5.69 \%$ of the isolates; 3 VFs ( $f i m H$ was present in all the combinations 
TABLE 3: The association of different VF genes with PGs.

\begin{tabular}{lccccc}
\hline & GR A & GR B1 & GR B2 & GR D & p-value \\
\hline fimH & $155(91.17 \%)$ & $204(94 \%)$ & $264(95.65 \%)$ & $116(94.30 \%)$ & 0.2873 \\
hlyD & $51(30 \%)$ & $72(33.17 \%)$ & $118(42.75 \%)$ & $7(2.53 \%)$ & $25(20.32 \%)$ \\
hlyA & $1(0.58 \%)$ & $3(1.38 \%)$ & $69(25 \%)$ & $14(11.38 \%)$ & 0.0196 \\
afaBC & $21(12.35 \%)$ & $43(19.81 \%)$ & $96(34.78 \%)$ & $13(10.56 \%)$ & 0.0010 \\
KpsMTII & $12(7.05 \%)$ & $72(33.17 \%)$ & $127(46.01 \%)$ & $9(7.31 \%)$ & $<0.0001$ \\
sfaDE & $13(7.64 \%)$ & $42(19.35 \%)$ & $32(11.59 \%)$ & $6(4.87 \%)$ & 0.00001 \\
cnf-1 & $2(1.17 \%)$ & $13(5.99 \%)$ & & 0.0005 \\
\hline
\end{tabular}

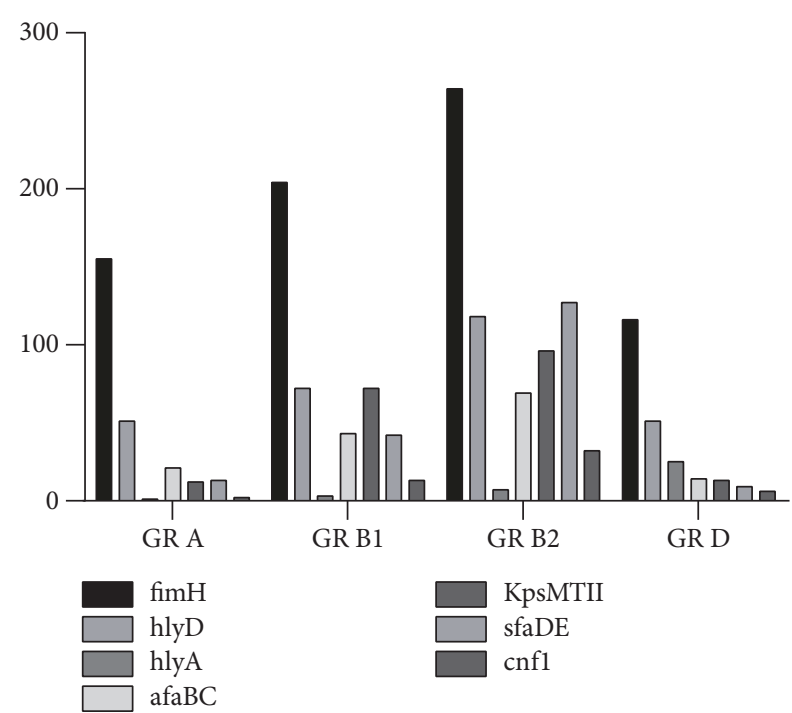

FIGURE 2: The distribution of VFs by PGs.

also) in $16.26 \%$; 2 VFs in $36.58 \%$; and one VFs $(37.39 \%$ of the isolates). The strains belonging to the PG A ( $n=170$ strains) revealed $4 \mathrm{VFs}$ ( $f m H, h l y D, s f a D E$, and $c n f-1$ ) in $1.76 \%$ of the isolates; $3 \mathrm{VFs}$ (fimH in all the combinations and $h l y D / a f a B C / k p s M T I I / s f a D E / c n f-1)$ in $7.64 \% ; 2$ VFs in $32.94 \%$; and one VFs (54.11\% from which fimH was revealed by $96.73 \%$ and $h l y D$ from $3.26 \%$ of the isolates). In case of PG B1 strains there were detected up to $5 \mathrm{VFs}$ ( $f m H$, $h l y D$, kpsMTII, sfaDE, cnf-1 orhlyA) in $1.84 \%$ of the isolates; 4 VFs (with fim $\mathrm{H}$ being revealed in all of them excepting one) in $8.29 \%$ of the isolates; 3 VFs in $20.27 \%$; 2 VFs in $35.48 \%$; and one VFs (32.71\% of the isolates). The strains classified in PG B2 demonstrated the presence of 6 VFs in $0.36 \%$ of the UPEC isolates; 5 VFs in $5.79 \%$; 4 VFs (fimH being revealed in all of them) in $18.47 \%$ of the isolates; 3 VFs in $25.36 \%$; 2 VFs in $28.62 \%$; and one VF in $19.92 \%$ of the isolates.

The strains classified in PG B1 and PG B2 were the most virulent ones, the number of strains carrying $>3 \mathrm{VFs}$ being significantly larger than that of strains belonging to PG A and PG D, where predominating the strains carrying less VFs $(\leq$ 3) $(\mathrm{p}<0.0001)$ (Figure 3).

Only few studies are reported in literature for CA-UPEC isolates. A study performed on Uruguayan children with UTIs revealed that $48.2 \%$ of the E. coli isolates belonged to PG D and $35.5 \%$ to PG B2, with the most frequent VFs being

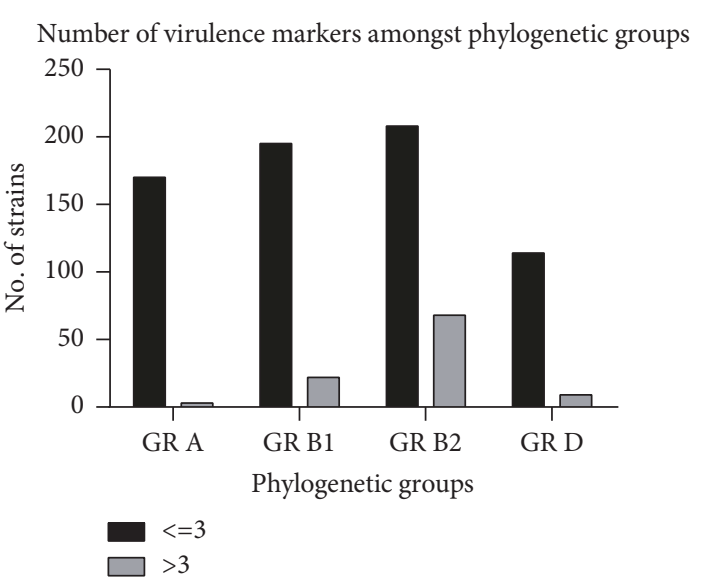

FIgURE 3: The distribution of the number of VFs among PGs.

kpsMTII and fimH [22]. Among 59 isolates of UPEC isolated in Pakistan from CA-UTIs, the PG B2 was the most frequent (50\%), followed by PG A, B1 (19\% each), and D (12\%). Isolates present in group D showed the highest number of VFs, among which the most frequent were hlyA (37\%), sfaDE (27\%), papC (24\%), cnf1 (20\%), eaeA (19\%), and afaBC3 (14\%) [23].

Of the UPEC adhesins, fimH, a type 1 fimbriae, has a crucial role in UPEC colonization in the bladder, which is required for the initiation of UTI [24]. E. coli afimbrial adhesin (Afa) encoded by afa gene has been reported in cases of pyelonephritis and recurring cystitis; another adhesin that acts as a virulence factor is S fimbrial adhesin, which is encoded by sfa genes [25]. Other very important virulence factors in UPEC strains, like toxins, mediating invasion, dissemination, and persistence of bacteria in host cells have been demonstrated [26]. The most important soluble virulence factor is $\alpha$-hemolysin (HlyA), which is encoded by the hly gene. Also, the cytotoxic necrotizing factor 1 (CNF1) is one of the most important virulence factors of $E$. coli involved in the development of an UTI. It has been revealed that $\alpha$-hemolysin and CNF1 mediate the release of iron from red blood cells, induce dysfunction of phagocytic cells, and exhibit direct cytotoxicity to the tissues [27]. Some authors report that the prevalence ratios of $s f a, h l y A$, and iron uptake genes were 2.2 to 3.5 times more prevalent among outpatients compared with inpatients UTI isolates [28].

According to the present observations, fim $\mathrm{H}$ gene had the highest frequency (93.90\%) among the tested VF genes, while 
TABLE 4: Virulence genes profiles and PGs in the investigated E. coli strains.

\begin{tabular}{lc}
\hline VF & Number (percentage) \\
\hline fimH & $739(93.90 \%)$ \\
hlyD & $349(44.34 \%)$ \\
afaBC & $301(38.24 \%)$ \\
$k p s M T I I$ & $257(32.65 \%)$ \\
sfaDE & $188(23.88 \%)$ \\
$h l y A$ & $98(12.45 \%)$ \\
$c n f-1$ & $61(7.75 \%)$ \\
$P G s$ & \\
$B 2$ & $276(35 \%)$ \\
$B 1$ & $216(27 \%)$ \\
$A$ & $173(22 \%)$ \\
$D$ & $122(16 \%)$ \\
\hline
\end{tabular}

cnf- 1 had the lowest one (7.75\%) (Table 4). Similar results have been revealed in Romania by Grosu et al. in 2017 [29] in E. coli strains isolated from the ambulatory sector of Central Laboratory Regina Maria hospital in Bucharest. The fimH gene was also reported to have a high prevalence $(>90 \%$ $100 \%$ ) among UPEC strains isolated in other countries, and some authors are stating that FimH could be used as a possible diagnostic marker and/or vaccine candidate [30-32]. The study of Tobasi et al. [28] performed on 156 UPEC isolated from symptomatic and asymptomatic UTI outpatients and inpatients revealed that fimH was present in all analyzed strains. Derakhshandeh et al., in 2015 [33], have reported that, from 85 UPEC clinical isolates, $65.9 \%$ belonged to phylogenetic group A, $17.6 \%$ belonged to $\mathrm{B} 2$, and $16.5 \%$ of the isolates were found to belong to group $\mathrm{D}$; fimH has also been reported with the highest frequency among the tested VF genes, while $c n f-1$ had the lowest one, similar to our results.

According to Rodriguez-Siek study in 2005 [34], most of UPEC causing UTI in human revealed capsule, the capsular antigen K1 being more often observed in UPEC. The capsule production in E. coli trains is mediated bykpsMT (encoding for K1 antigen) and kpsMTII genes [35]. A study performed on a total of $194 \mathrm{E}$. coli strains isolated in Mexico from CA-UTIs has shown that kpsMT was the most frequently occurring virulence gene among the UPEC strains (92.2\% strains), the fim gene being also recorded with a high positivity rate (61.3\%) [36]. Farajzadah et al., in 2018 [16], have reported that, among the 232 analyzed UPEC strains, the most frequently encountered PG was D (58\%) responsible for majority of nosocomial (64.7\%) and community (48.4\%) acquired infections with the largest panel of VF genes, including kpsMT (23\%) and cnf (29.6\%). Ochoa et al., in 2016 [37], have found that, among 500 UPEC clinical strains, 103 were MDR-UPEC strains and mainly associated with the phylogenetic groups D (54.87\%) and B2 (39.02\%) with a high percentage of positivity for $f i m H$, an iron uptake gene (chuA), and a toxin gene $(h l y A)$.

We have also investigated the potential correlations between the PG and VF genes and the presence of different resistance phenotypes (multiple logistic regression) or ESBL genes (chi square). Our results pointed out that there is no statistically significant correlation between PG and VF and the presence of certain resistance phenotypes, which suggests that there are no particular clones associated with UTIs in Romania. On the other hand, the presence of one or two ESBL genes was significantly associated ( $\mathrm{p}=0.0024)$ with PGs A and D.

In France, UPEC isolated from UTIs belonged more frequently to phylotypes $\mathrm{B} 2$ and $\mathrm{D}$, the strains susceptible to ciprofloxacin harboring specific VFs profiles, more extended in comparison with the ciprofloxacin-resistant strains [38]. Another study performed on 146 E. coli strains isolated from cystitis and pyelonephritis in Turkey investigated the relationship among PGs and various adhesion virulence genes. The $s f a / f o c D E$ genes were more frequent in ampicillin, amikacin, gentamicin, nalidixic acid, norfloxacin, cefuroxime, ceftriaxone, cefazolin, cefotaxime, ciprofloxacin and cotrimoxazole susceptible and extended spectrum $\beta$ lactamase (ESBL), and multidrug resistance (MDR) negative isolates. fimH was more common in amoxicillinclavulanic acid susceptible isolates. The afa gene was more frequent in resistant isolates than in susceptible ones [39].

\section{Conclusions}

To the best of our knowledge, this is the first study performed on a significant number of $E$. coli strains isolated from outpatients with community-acquired urinary tract infections in Bucharest, Romania, aiming to investigate the correlations among the phylogenetic group, resistance, and virulence profiles of CA-UPEC strains. The analyzed strains exhibited resistance rates ranging from $47.52 \%$ for ampicillin to $14.86 \%$ for levofloxacin, $35.19 \%$ were MDR phenotype, and $9.03 \%$ were ESBL producers. The fimH gene was the most frequent (93.90\%), followed by hlyD (44.34\%); afaBC (38.24\%); KpsMTII (32.65\%); sfaDE (23.88\%); hlyA(12.45\%); and $c n f-1(7.75 \%)$. The phylogenetic group distribution was different, depending on the resistance phenotype. Overall, our findings showed that the CA-UPEC strains isolated from outpatients in Bucharest, Romania, belong predominantly to group B2 and $>90 \%$ harbor the fimH gene. High MDR resistance rates were observed, the ESBL phenotype being associated with PGs A and D. The most extended VF profiles were encountered in CA-UPEC strains classified in the PGs $\mathrm{B} 1$ and $\mathrm{B} 2$. The obtained results highlight the importance of this type of studies for improving the epidemiological surveillance and the therapeutic or prophylactic management of the respective infections, in the context of antibiotic resistance emergence.

\section{Methods}

The study was conducted on a total of 787 strains isolated during one month in 2018 from outpatients visiting Synevo Central Laboratory, Medicover, in Bucharest, Romania. The 
TABLE 5: Primers sequences used in simplex and multiplex PCR assays for genes encoding BLSE.

\begin{tabular}{llccr}
\hline The gene & Primer & Nucleotide sequence & $\begin{array}{c}\text { Amplification } \\
\text { size }\end{array}$ & References \\
\hline$b l a_{\mathrm{TEM}}$ & TEM-F & 5'-ATGAGTTTTCAACATTTTCG-3' & \multirow{2}{*}{861} & Eftekar et al., 2005 [40] \\
\hline$b l a_{\mathrm{SHV}}$ & TEM-R & 5'-TTACCAATGCTTAATCAG TG-3' & \multirow{2}{*}{ Naas et al., 1999 [41] } \\
\hline$b l a_{\text {CTX-M }}$ & SHV-F & 5'-GCCCTCACTCAAGGATGTAT-3' & \multirow{2}{*}{ Israil et al., 2013 [42] } \\
\hline
\end{tabular}

Table 5 is partially reproduced from Grosu et al. 2017 [underthe Creative Commons Attribution License/public domain].

TABLE 6: Primers sequences used in simplex and multiplex PCR assays for virulence genes.

\begin{tabular}{|c|c|c|c|}
\hline The gene & Primer & $\begin{array}{c}\text { Amplification size } \\
\text { and } \\
\text { Tm }\end{array}$ & References \\
\hline chuA & $\begin{array}{l}\text { F: } 5^{\prime} \text {-GACGAACCAACGGTCAGGAT-3' } \\
\text { R: } 5^{\prime} \text {-TGCCGCCAGTACCAAAGACA-3' }\end{array}$ & $\begin{array}{c}279 \text { bp } \\
\text { (multiplex) } 55^{\circ} \mathrm{C}\end{array}$ & Clermont et al., 2000 \\
\hline$y j a A$ & $\begin{array}{l}\text { F: 5'-TGAAGTGTCAGGAGACGCTG-3' } \\
\text { R: } 5^{\prime} \text {-ATGGAGAATGCGTTCCTCAAC-3' }\end{array}$ & $\begin{array}{c}\text { 211bp } \\
\text { (multiplex) } 55^{\circ} \mathrm{C}\end{array}$ & Clermont et al., 2000 \\
\hline TspE4C2 & $\begin{array}{l}\text { F: } 5^{\prime} \text {-GAGTAATGTCGGGGCATTCA-3' } \\
\text { R:5'-CGCGCCAACAAAGTATTACG-3' }\end{array}$ & $\begin{array}{l}152 \mathrm{bp} \text { (multiplex) } \\
55^{\circ} \mathrm{C}\end{array}$ & Clermont et al., 2000 \\
\hline hlyD & $\begin{array}{l}\text { F: } 5^{\prime} \text { - CTCCGGTACGTGAAAAGGAC-3' } \\
\text { R: } 5^{\prime} \text {-GCCCTGATTACTGAAGCCTG-3' }\end{array}$ & $\begin{array}{c}904 \mathrm{bp} \\
55^{\circ} \mathrm{C}\end{array}$ & Rodrigues-Sike et al., 2005 \\
\hline kpsMTII & $\begin{array}{l}\text { F: } 5^{\prime}-\text { GCG CAT TTG CTG ATA CTG TTG-3' } \\
\text { R: 5'-CAT CAG ACG ATA AGC ATG AGC A-3' }\end{array}$ & $\begin{array}{r}272 \mathrm{bp} \\
60^{\circ} \mathrm{C}\end{array}$ & Johnson et al., 2005 [43] \\
\hline hlyA & $\begin{array}{l}\text { F:5'-AACAAGGATAAGCACTGT TCTGGC T-3' } \\
\text { R:5'-ACCATATAAGCGGTCATT CCC GTC A-3' }\end{array}$ & $\begin{array}{l}1,177 \mathrm{bp} \\
60^{\circ} \mathrm{C}\end{array}$ & Yamamoto et al., 1995 [44] \\
\hline$s f a D / E$ & $\begin{array}{l}\text { F:5'-CGGAGGAGTAATTACAAACCTGGCA -3' } \\
\text { R: 5' - CTCCGGAGAACTGGGTG ATCTTA C-3' }\end{array}$ & $\begin{array}{c}408 \mathrm{bp} \\
60^{\circ} \mathrm{C}\end{array}$ & Blanco et al., 1997 [45] \\
\hline fimH & $\begin{array}{l}\text { F: } 5^{\prime} \text {-TGC AGA ACG GAT AAG CCG TGG - } 3^{\prime} \\
\text { R: } 5^{\prime} \text { - GCA GTC ACC TGC CCT CCG GTA - } 3^{\prime}\end{array}$ & $\begin{array}{r}508 \mathrm{bp} \\
63^{\circ} \mathrm{C}\end{array}$ & Rodrigues-Sike et al., 2005 \\
\hline$a f a B C$ & $\begin{array}{c}\text { F: 5'-GCTGGGCAGCAAACTGATAACTCTC -3' } \\
\text { R:5'CATCAAGCTGTTTGTTCGTCCGCCG-3 }\end{array}$ & $\begin{array}{r}793 \mathrm{bp} \\
63^{\circ} \mathrm{C}\end{array}$ & Blanco et al., 1997 \\
\hline$c n f-1$ & $\begin{array}{c}\text { F: } 5^{\prime} \text { - GAA CTT ATT AAG GAT AGT-3' } \\
\text { R: } 5^{\prime} \text {-CAT TAT TTA TAA CGC TG-3' }\end{array}$ & $\begin{array}{r}543 \mathrm{~kb} \\
40^{\circ} \mathrm{C}\end{array}$ & Blanco et al., 1997 \\
\hline
\end{tabular}

Table 6 is reproduced from Grosu et al. 2017 [under the Creative Commons Attribution License/public domain].

strains identification was performed using the MALDI TOF system and the susceptibility patterns were tested by Microscan according to CLSI 2018 guidelines.

4.1. DNA Extraction and Molecular Detection. The genetic support of the antibiotic resistance (ESBLs) and virulence markers was investigated by simplex and multiplex PCR, using a reaction mix of $20 \mu \mathrm{l}$ (PCR Master Mix 2x, Thermo Scientific containing $\mathrm{MgCl}_{2} 1.2 \mathrm{mM}$, dNTP $2 \mu \mathrm{M}$ DNA $0.2 \mathrm{U}$ Taq-pol $1 \mathrm{x}$ and Reaction buffer until the final volume) to which the primers at $0.5 \mu \mathrm{M}$ and and $1 \mu \mathrm{l}$ of bacterial DNA extracted by an adapted alkaline extraction method. In this purpose, 1-5 colonies of bacterial cultures were suspended in $1.5 \mathrm{ml}$ tubes containing $20 \mu \mathrm{l}$ solution of $0.05 \mathrm{M} \mathrm{NaOH}$ (sodium hydroxide) and $0.25 \%$ SDS (sodium dodecyl sulphate). The amplification program was conducted under the following conditions: $94^{\circ} \mathrm{C}, 10 \mathrm{~min} ; 94^{\circ} \mathrm{C}, 30 \mathrm{~s} ; 52^{\circ} \mathrm{C}, 40 \mathrm{~s}, 36$ cycles; $72^{\circ} \mathrm{C} 50 \mathrm{~s} ; 72^{\circ} \mathrm{C} 5 \mathrm{~min}$.

Bacterial DNA were subjected to simplex PCR targeting the chuA gene, the yjaA gene, and an unspecified DNA fragment termed TspE4.C2, as described previously [17]. Isolates were classified as belonging to one of the four phylogenetic groups A, B1, B2, or D. The sequence of the primers used in PCR experiments, the amplicon size obtained and the sources are presented in Tables 5 and 6.

\section{Statistical Analysis}

Statistical analysis was carried out using chi square and chi square test for trend tests using Graph Pad Prism version 8.0.1 (244) and multiple logistic regression using Stats Direct version 3 . For all statistical tests, $\mathrm{p}$ values $<0.05$ were considered significant. 


\section{Data Availability}

All data analyzed or generated during this study are included in this published article.

\section{Conflicts of Interest}

The authors declare that they have no conflicts of interest.

\section{Authors' Contributions}

Violeta Corina Cristea, Ioan Mircea Popa, and Mariana Carmen Chifiriuc designed the study and corrected the manuscript, Violeta Corina Cristea performed the isolation and identification, Irina Gheorghe, Laura Ioana Popa, Ilda Czobor Barbu, Bogdan Ispas, Georgiana Alexandra Grigore, Alexandra Velican, Luminita Marutescu, Marcela Popa, and Ilda Czobor Barbu performed the molecular analyses, Gabriela Loredana Popa has contributed to the design of the study, analysis of the results, drafting and the correction of the manuscript, Irina Gheorghe, and Ilda Czobor Barbu performed the statistical analysis and drafted the paper. Violeta Corina Cristea, Irina Gheorghe, Ilda Czobor Barbu, Gabriela Loredana Popa, Alexandra Velican, Luminita Marutescu, Marcela Popa, Mariana Carmen Chifiriuc, and Ioan Mircea Popa have equally contributed to this paper as main authors.

\section{Acknowledgments}

The authors gratefully acknowledge the financial support of the research projects PNIII-P4-ID-PCCF-2016-0114 and PD $113 / 2018$.

\section{References}

[1] B. Foxman, "Epidemiology of urinary tract infections: incidence, morbidity, and economic costs," Disease-a-Month, vol. 49, no. 2, pp. 53-70, 2003.

[2] J.-P. Lavigne, H. Marchandin, J. Delmas et al., "CTX-M beta-lactamase-producing Escherichia coli in French hospitals: prevalence, molecular epidemiology, and risk factors," Journal of Clinical Microbiology, vol. 45, no. 2, pp. 620-626, 2007.

[3] M. Arisoy, A. Y. Rad, A. Akin, and N. Akar, "Relationship between susceptibility to antimicrobials and virulence factors in paediatric Escherichia coli isolates," International Journal of Antimicrobial Agents, vol. 31, no. 1, pp. 4-8, 2008.

[4] M. E. Poey and M. Laviña, "Integrons in uropathogenic Escherichia coli and their relationship with phylogeny and virulence," Microbial Pathogenesis, vol. 77, pp. 73-77, 2014.

[5] H. Momtaz, A. Karimian, M. Madani et al., "Uropathogenic Escherichia coli in Iran: serogroup distributions, virulence factors and antimicrobial resistance properties," Annals of Clinical Microbiology and Antimicrobials, vol. 12, no. 1, article 8, 2013.

[6] T. L. Griebling, "Urinary Tract Infections in Women," in Urologic Diseases in America, M. S. Litwin and C. S. Saigal, Eds., pp. 587-620, US Department of Health and Human Services, Public Health Service, National Institutes of Health, National Institute of Diabetes and Digestive and Kidney Diseases, Washington, DC, USA, 2007.
[7] K. W. Yun, H. Y. Kim, H. K. Park, W. Kim, and I. S. Lim, "Virulence factors of uropathogenic Escherichia coli of urinary tract infections and asymptomatic bacteriuria in children," Journal of Microbiology, Immunology and Infection, vol. 47, no. 6, pp. 455-461, 2014.

[8] V. Merle, J.-M. Germain, H. Bugel et al., "Nosocomial urinary tract infections in urologic patients: Assessment of a prospective surveillance program including 10,000 patients," European Urology, vol. 41, no. 5, pp. 483-489, 2002.

[9] K. Wada, R. Kariyama, R. Mitsuhata et al., "Experimental and clinical studies on fluoroquinolone-insusceptible Escherichia coli isolated from patients with urinary tract infections from 1994 to 2007," Acta Medica Okayama, vol. 63, no. 5, pp. 263-272, 2009.

[10] J. Medina-Polo, A. Arrébola-Pajares, S. Pérez-Cadavid et al., "Extended-spectrum beta-lactamase-producing bacteria in a urology ward: epidemiology, risk factors and antimicrobial susceptibility patterns," Urologia Internationalis, vol. 95, no. 3, pp. 288-292, 2015.

[11] O. Clermont, J. K. Christenson, E. Denamur, and D. M. Gordon, "The Clermont Escherichia coli phylo-typing method revisited: improvement of specificity and detection of new phylo-groups," Environmental Microbiology Reports, vol. 5, no. 1, pp. 58-65, 2013.

[12] L. C. Da Silva, A. C. De Mello Santos, and R. M. Silva, "Uropathogenic Escherichia coli pathogenicity islands and other ExPEC virulence genes may contribute to the genome variability of enteroinvasive E. coli," BMC Microbiology, vol. 17, no. 1, article no 68, 2017.

[13] J. R. Johnson, B. Johnston, C. Clabots, M. A. Kuskowski, and M. Castanheira, "Escherichia coli sequence type ST131 as the major cause of serious multidrug-resistant E. coli infections in the United States," Clinical Infectious Diseases, vol. 51, no. 3, pp. 286-294, 2010.

[14] G. J. da Silva and N. Mendonça, "Association between antimicrobial resistance and virulence in Escherichia coli," Virulence, vol. 3, no. 1, pp. 18-28, 2012.

[15] G. Peirano and J. D. D. Pitout, "Molecular epidemiology of Escherichia coli producing CTX-M beta-lactamases: the worldwide emergence of clone ST131 O25:H4," International Journal of Antimicrobial Agents, vol. 35, no. 4, pp. 316-321, 2010.

[16] A. Farajzadah Sheikh, S. Aslani, S. A. Hosseini et al., "Phylotyping based virulence associated genes and drug susceptibility of Uropathogenic Escherichia coli in urinary tract infection patients," Journal of Microbiology and Biotechnology, 2018.

[17] O. Clermont, S. Bonacorsi, and E. Bingen, "Rapid and simple determination of the Escherichia coli phylogenetic group," Applied and Environmental Microbiology, vol. 66, no. 10, pp. 4555-4558, 2000.

[18] M. Doumith, M. J. Day, R. Hope, J. Wain, and N. Woodford, "Improved multiplex PCR strategy for rapid assignment of the four major Escherichia coli phylogenetic groups," Journal of Clinical Microbiology, vol. 50, no. 9, pp. 3108-3110, 2012.

[19] M. T. Nüesch-Inderbinen, M. Baschera, K. Zurfluh, H. Hächler, H. Nüesch, and R. Stephan, "Clonal diversity, virulence potential and antimicrobial resistance of escherichia coli causing community acquired urinary tract infection in Switzerlan," Frontiers in Microbiology, vol. 8, 2017.

[20] A.-P. Magiorakos, A. Srinivasan, R. B. Carey et al., "Multidrugresistant, extensively drug-resistant and pandrug-resistant bacteria: an international expert proposal for interim standard 
definitions for acquired resistance," Clinical Microbiology and Infection, vol. 18, no. 3, pp. 268-281, 2012.

[21] M. Yahiaoui, F. Robin, R. Bakour, M. Hamidi, R. Bonnet, and Y. Messai, "Antibiotic resistance, virulence, and genetic background of community-acquired uropathogenic Escherichia coli from Algeria," Microbial Drug Resistance, vol. 21, no. 5, pp. 516526, 2015.

[22] L. Robino, V. García-Fulgueiras, L. Araujo, G. Algorta, M. C. Pírez, and R. Vignoli, "Urinary tract infection in Uruguayan children: Aetiology, antimicrobial resistance and uropathogenic Escherichia coli virulotyping," Journal of Global Antimicrobial Resistance, vol. 2, no. 4, pp. 293-298, 2014.

[23] S. Bashir, A. Haque, Y. Sarwar, A. Ali, and M. I. Anwar, "Virulence profile of different phylogenetic groups of locally isolated community acquired uropathogenic E. coli from Faisalabad region of Pakistan," Annals of Clinical Microbiology and Antimicrobials, vol. 11, article 23, 2012.

[24] G. C. Ulett, M. Totsika, K. Schaale, A. J. Carey, M. J. Sweet, and M. A. Schembri, "Uropathogenic Escherichia coli virulence and innate immune responses during urinary tract infection," Current Opinion in Microbiology, vol. 16, no. 1, pp. 100-107, 2013.

[25] A. L. Servin, "Pathogenesis of Afa/Dr diffusely adhering Escherichia coli," Clinical Microbiology Reviews, vol. 18, no. 2, pp. 264-292, 2005.

[26] J. Bien, O. Sokolova, and P. Bozko, "Role of uropathogenic escherichia coli virulence factors in development of urinary tract infection and kidney damage," International Journal of Nephrology, vol. 2012, Article ID 681473, 15 pages, 2012.

[27] M. Pourzare, S. Derakhshan, and D. Roshani, "Distribution of uropathogenic virulence genes in escherichia coli isolated from children with urinary tract infection in Sanandaj, Iran," Archives of Pediatric Infectious Diseases, vol. 5, no. 3, Article ID e41995, 2017.

[28] M. Tabasi, M. R. A. Karam, M. Habibi, E. Mostafavi, and S. Bouzari, "Genotypic characterization of virulence factors in Escherichia coli isolated from patients with acute cystitis, pyelonephritis and asymptomatic bacteriuria," Journal of Clinical and Diagnostic Research, vol. 10, no. 12, pp. DC01-DC07, 2016.

[29] M. Grosu, M. Chelariu, I. Gheorghe et al., "Genotypic characterization of virulence and resistance markers in ESBL positive E. coli strains isolated from ambulatory urinary tract infections," Romanian Biotechnological Letters, vol. 22, 2017.

[30] U. B. Maheswari, S. Palvai, P. R. Anuradha, and N. Kammili, "Hemagglutination and biofilm formation as virulence markers of uropathogenic Escherichia coli in acute urinary tract infections and urolithiasis," Indian Journal of Urology, vol. 29, no. 4, pp. 277-281, 2013.

[31] Y. Millán, E. Hernández, B. Millán, and M. Araque, "Distribution of phylogenetic groups and virulence factors in CTX-M$15 \beta$-lactamaseproducing uropathogenic escherichia coli strains isolated from patients in the community of mérida, Venezuela," Revista Argentina de Microbiología, vol. 46, no. 3, pp. 175-181, 2014.

[32] Z. Hojati, B. Zamanzad, M. Hashemzadeh, R. Molaie, and A. Gholipour, "The FimH gene in uropathogenic escherichia coli strains isolated from patients with urinary tract infection," Jundishapur Journal of Microbiology, vol. 8, no. 2, Article ID e17520, 2015.

[33] A. Derakhshandeh, R. Firouzi, M. Motamedifar et al., "Virulence characteristics and antibiotic resistance patterns among various phylogenetic groups of uropathogenic Escherichia coli isolates," Japanese Journal of Infectious Diseases, vol. 68, no. 5, pp. 428-431, 2015.

[34] K. E. Rodriguez-Siek, C. W. Giddings, C. Doetkott, T. J. Johnson, M. K. Fakhr, and L. K. Nolan, "Comparison of Escherichia coli isolates implicated in human urinary tract infection and avian colibacillosis," Microbiology, vol. 151, no. 6, pp. 2097-2110, 2005.

[35] F. Bert, J. R. Johnson, B. Ouattara et al., "Genetic diversity and virulence profiles of Escherichia coli isolates causing spontaneous bacterial peritonitis and bacteremia in patients with cirrhosis," Journal of Clinical Microbiology, vol. 48, no. 8, pp. 2709-2714, 2010.

[36] G. L. Paniagua-Contreras, E. Monroy-Pérez, J. R. RodríguezMoctezuma, P. Domínguez-Trejo, F. Vaca-Paniagua, and S. Vaca, "Virulence factors, antibiotic resistance phenotypes and O-serogroups of Escherichia coli strains isolated from community-acquired urinary tract infection patients in Mexico," Journal of Microbiology, Immunology and Infection, vol. 50, no. 4, pp. 478-485, 2015.

[37] S. A. Ochoa, A. Cruz-Córdova, V. M. Luna-Pineda et al., "Multidrug - and extensively drug-resistant uropathogenic escherichia coli clinical strains: phylogenetic groups widely associated with integrons maintain high genetic diversity," Frontiers in Microbiology, vol. 7, article no 2042, pp. 1-12, 2016.

[38] J.-P. Lavigne, F. Bruyère, L. Bernard et al., "Resistance and virulence potential of uropathogenic Escherichia coli strains isolated from patients hospitalized in urology departments: A French prospective multicentre study," Journal of Medical Microbiology, vol. 65, no. 6, pp. 530-537, 2016.

[39] D. K. Er, D. Dundar, H. Uzuner, and A. Osmani, "Relationship between phylogenetic groups, antibiotic resistance and patient characteristics in terms of adhesin genes in cystitis and pyelonephritis isolates of Escherichia coli," Microbial Pathogenesis, vol. 89, pp. 188-194, 2017.

[40] R. F. Eftekar, S. M. Hosseini-Mazinani, S. Ghandili, M. Hamraz, and S. Zamani, "PCR detection of plasmid mediated TEM, SHV and AmpC $\beta$-lactamases in community and nosocomial urinary isolates of Escherichia coli," Iranian Journal of Biotechnology, vol. 3, no. 1, pp. 48-54, 2005.

[41] T. Naas, L. Philippon, L. Poirel, E. Ronco, and P. Nordmann, "An SHV-derived extended-spectrum $\beta$-lactamase in Pseudomonas aeruginosa," Antimicrobial Agents and Chemotherapy, vol. 43, no. 5, pp. 1281-1284, 1999.

[42] A. Israil, C. Chifiriuc, G. Palade, and A. Cotar, "Clinical and bacteriological aspects of bacterial infections associated to abdominal surgical emergencies," Ars Docenti Publ House, vol. $150,2013$.

[43] J. R. Johnson, M. A. Kuskowski, A. Gajewski et al., "Extended virulence genotypes and phylogenetic background of Escherichia coli isolates from patients with cystitis, pyelonephritis, or prostatitis," The Journal of Infectious Diseases, vol. 191, no. 1, pp. 46-50, 2005.

[44] S. Yamamoto, A. Terai, K. Yuri et al., "Detection of urovirulence factors in Escherichia coli by multiplex polymerase chain reaction," FEMS Immunology \& Medical Microbiology, vol. 12, no. 2, pp. 85-90, 1995.

[45] M. Blanco, J. E. Blanco, M. P. Alonso et al., "Detection of pap, sfa and afa adhesin-encoding operons in uropathogenic Escherichia coli strains: Relationship with expression of adhesins and production of toxins," Research in Microbiology, vol. 148, no. 9, pp. 745-755, 1997. 


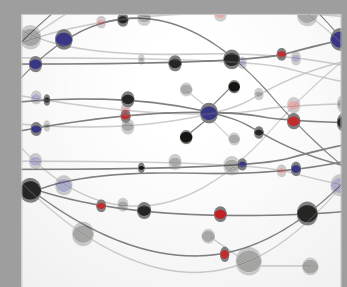

The Scientific World Journal
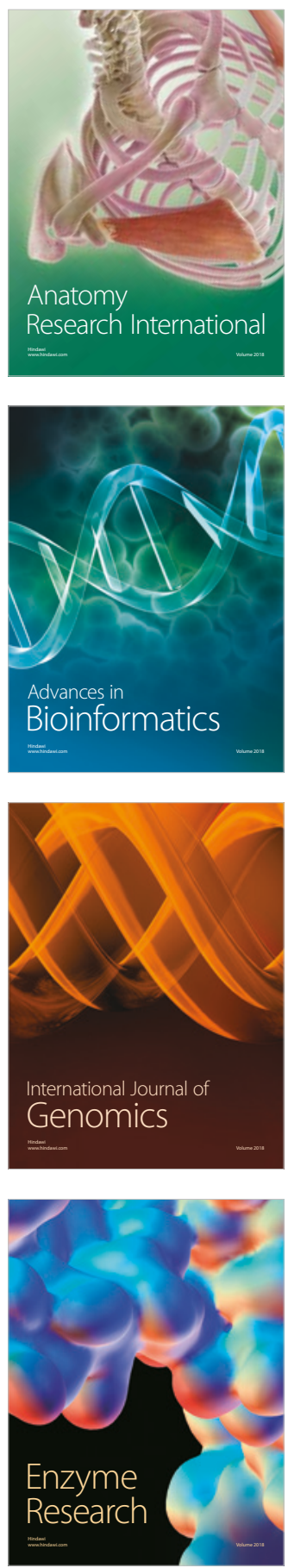
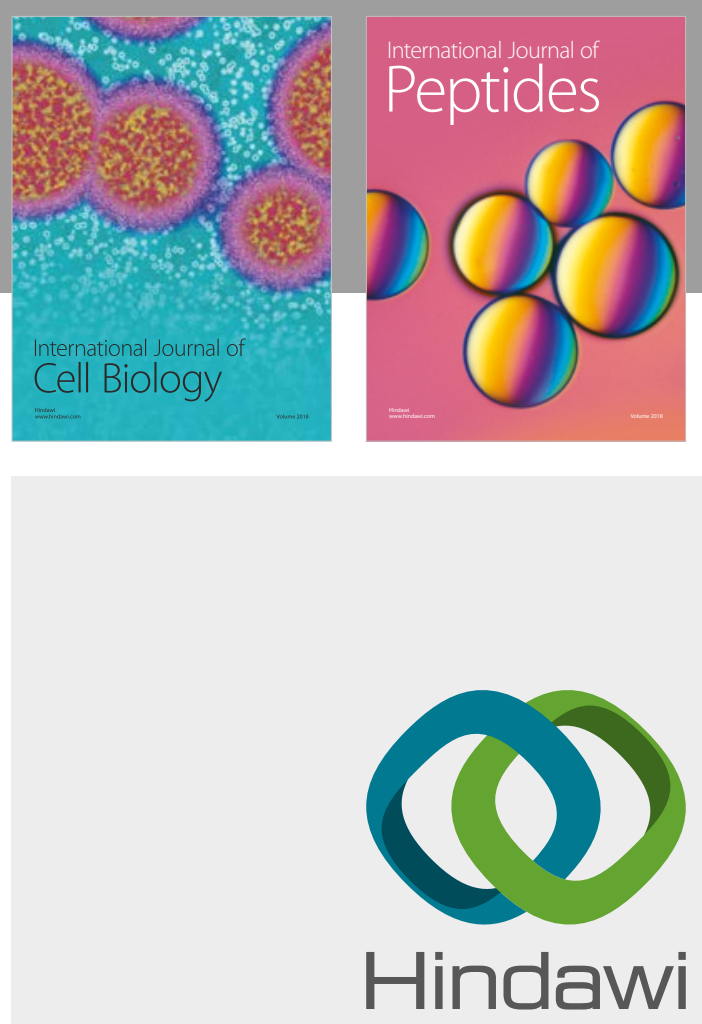

Submit your manuscripts at

www.hindawi.com
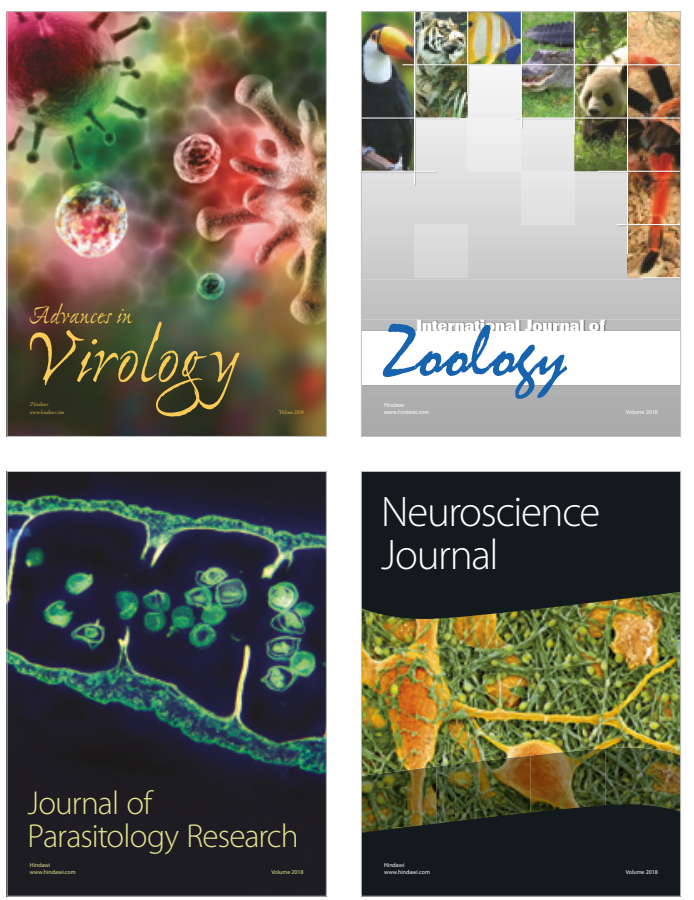
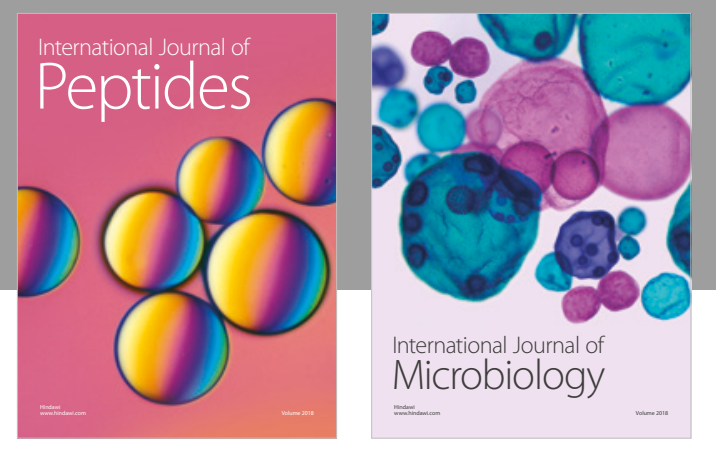

nternational Journal of Microbiology
Journal of
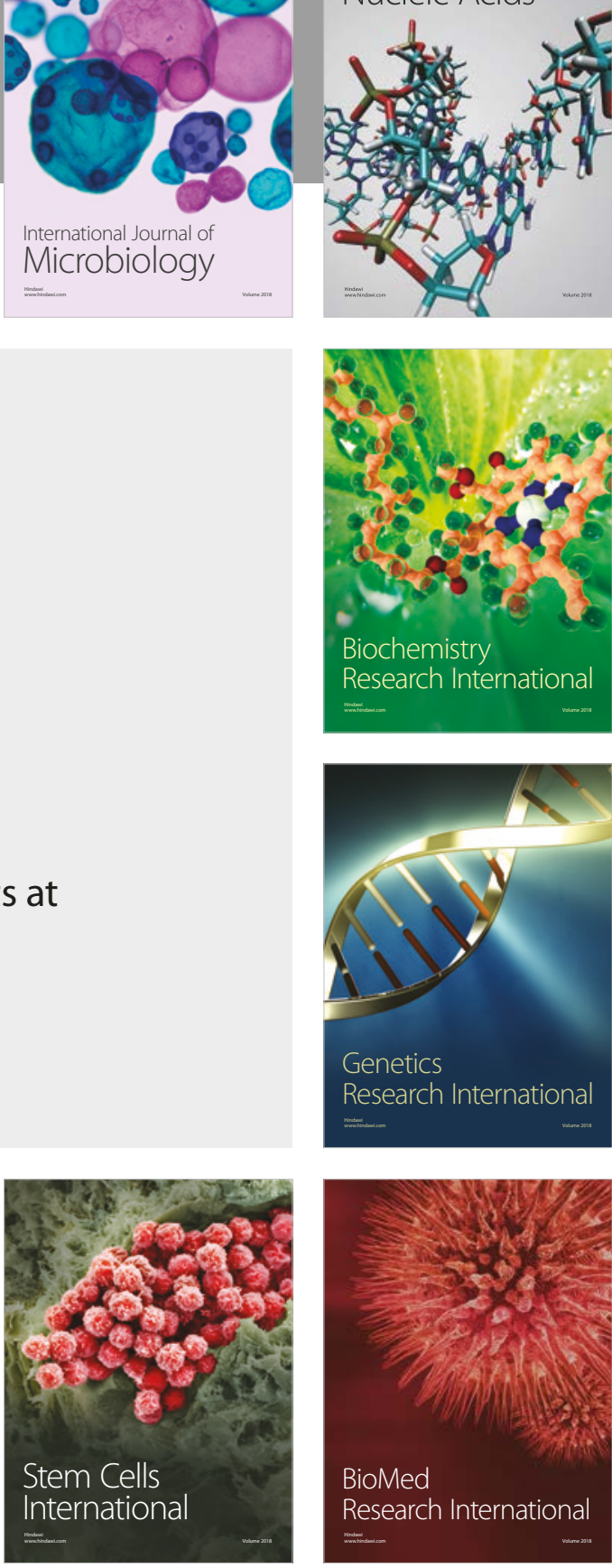
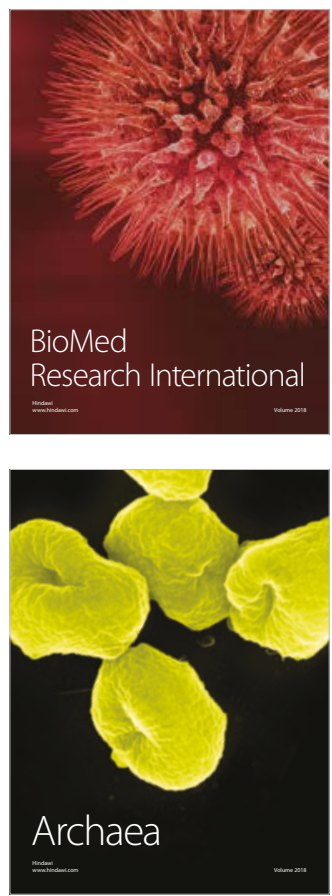